\title{
The effect of oat $\beta$-glucan on postprandial blood glucose and insulin responses: a systematic review and meta-analysis
}

\author{
Andreea Zurbau ${ }^{1,2,3} \cdot$ Jarvis C. Noronha' ${ }^{1}$ Tauseef A. Khan $\mathbb{1}^{2,3} \cdot$ John L. Sievenpiper ${ }^{2,3,4,5,6}$. \\ Thomas M. S. Wolever $\mathbb{B}^{1}$
}

Received: 27 August 2020 / Revised: 18 January 2021 / Accepted: 28 January 2021 / Published online: 19 February 2021

(c) The Author(s) 2021. This article is published with open access

\begin{abstract}
To determine the effect of oat $\beta$-glucan (OBG) on acute glucose and insulin responses and identify significant effect modifiers we searched the MEDLINE, EMBASE, and Cochrane databases through October 27, 2020 for acute, crossover, controlled feeding trials investigating the effect of adding OBG (concentrate or oat-bran) to carbohydrate-containing testmeals compared to comparable or different carbohydrate-matched control-meals in humans regardless of health status. The primary outcome was glucose incremental area-under-the-curve (iAUC). Secondary outcomes were insulin iAUC, and glucose and insulin incremental peak-rise (iPeak). Two reviewers extracted the data and assessed risk-of-bias and certaintyof-evidence (GRADE). Data were pooled using generic inverse-variance with random-effects model and expressed as ratioof-means with [95\% CIs]. We included 103 trial comparisons $(N=538)$. OBG reduced glucose iAUC and iPeak by $23 \%$ $(0.77[0.74,0.81])$ and $28 \%(0.72[0.64,0.76])$ and insulin by $22 \%(0.78[0.72,0.85])$ and $24 \%(0.76[0.65,0.88])$, respectively. Dose, molecular-weight, and comparator were significant effect modifiers of glucose iAUC and iPeak. Significant linear dose-response relationships were observed for all outcomes. OBG molecular-weight $>300 \mathrm{~kg} / \mathrm{mol}$ significantly reduced glucose iAUC and iPeak, whereas molecular-weight $<300 \mathrm{~kg} / \mathrm{mol}$ did not. Reductions in glucose iAUC ( 27 vs $20 \%, p=0.03$ ) and iPeak (39 vs $25 \%, p<0.01$ ) were significantly larger with different vs comparable control-meals. Outcomes were similar in participants with and without diabetes. All outcomes had high certainty-of-evidence. In conclusion, current evidence indicates that adding OBG to carbohydrate-containing meals reduces glycaemic and insulinaemic responses. However, the magnitude of glucose reduction depends on OBG dose, molecular-weight, and the comparator.
\end{abstract}

These authors contributed equally: Andreea Zurbau, Jarvis C. Noronha

Supplementary information The online version contains supplementary material available at https://doi.org/10.1038/s41430021-00875-9.

Thomas M. S. Wolever

twolever@inquis.com

1 INQUIS Clinical Research Ltd. (formerly GI Labs), Toronto, ON, Canada

2 Toronto 3D Knowledge Synthesis and Clinical Trials Unit, Clinical Nutrition and Risk Factor Modification Centre, St. Michael's Hospital, Toronto, ON, Canada

3 Department of Nutritional Sciences, Temerty Faculty of Medicine,

\section{Introduction}

$\beta$-glucan, a viscous soluble dietary fibre found naturally in oats and barley, has a number of potentially beneficial physiological effects which include reducing both postprandial glycaemic responses (PPGR) [1] and serum cholesterol [2-4]. The ability of $\beta$-glucan to reduce PPGR was established by a European Food Safety Authority (EFSA) Panel review that concluded that $4 \mathrm{~g}$ of either oat $\beta$-glucan

University of Toronto, Toronto, ON, Canada

4 Department of Medicine, Temerty Faculty of Medicine, University of Toronto, Toronto, ON, Canada

5 Division of Endocrinology and Metabolism, Department of Medicine, St. Michael's Hospital, Toronto, ON, Canada

6 Li Ka Shing Knowledge Institute, St. Michael's Hospital, Toronto, ON, Canada 
(OBG) or barley $\beta$-glucan (BBG) per $30 \mathrm{~g}$ of available carbohydrates (avCHO) is required to obtain a consistent reduction in PPGR [5]. A subsequent review confirmed this by showing that $\mathrm{OBG}$ and BBG significantly reduced PPGR in all studies that used doses $>4 \mathrm{~g} / 30 \mathrm{~g}$ avCHO [1]. However, we found that adding $1.7 \mathrm{~g}$ or $2.5 \mathrm{~g} \mathrm{OBG} / 30 \mathrm{~g}$ avCHO to instant oats or muffin significantly reduced glucose iAUC by $16 \%$ [6] and 24\% [7], respectively. Thus, the amount of OBG required to obtain a clinically meaningful reduction in PPGR is not clear. The estimate of $4 \mathrm{~g}$ OBG/30 $\mathrm{g}$ avCHO is imprecise because the previous reviews did not address the confounding effects of $\beta$-glucan source and molecular weight (MW) and their results are not expressed in such a way that clinical relevance can be assessed.

Both EFSA [5] and Tosh [1] included studies using oats or barley grains, flakes or flour as sources of $\beta$-glucan. This is a problem because each gram of $\beta$-glucan in oats and barley, respectively, is accompanied by $\sim 13 \mathrm{~g}$ and $\sim 11 \mathrm{~g}$ avCHO whose effect on glucose and insulin responses is influenced to a large extent by cooking and processing [8,9]. Thus, the inclusion of whole grains, flakes and flour confounds the effect of $\beta$-glucan in potentially unpredictable ways. Furthermore, since BBG differs from OBG with respect to the ratio of $\beta-(1 \rightarrow 3)$ to $\beta$ - $(1 \rightarrow 4)$ linkages, $\mathrm{MW}$, solubility and conformation [10], the effects of purified OBG on PPGR may differ from those of purified BBG. This is supported by the studies cited by Tosh [1] that used purified $\beta$-glucan or $\beta$-glucan concentrates (OBG, $n=41$; BBG, $n=$ 10). Although all $\beta$-glucan doses $>4 \mathrm{~g} / 30 \mathrm{~g}$ avCHO (OBG, $n=18$ BBG, $n=1$ ) significantly reduced PPGR, smaller doses reduced PPGR more often with OBG than BBG, 15 of $23(65 \%)$ vs 2 of $7\left(22 \%, \chi^{2} p=0.028\right)$. Therefore, we excluded studies using BBG from this analysis.

The MW and dose of OBG have independent effects on PPGR [7]. EFSA [5] did not consider MW, and by excluding treatments where the $\beta$-glucan MW had been deliberately reduced to $<250,000 \mathrm{~g} / \mathrm{mol}$, Tosh [1] was unable to assess the effect of MW on PPGR.

Tosh [1] found that $4 \mathrm{~g} \beta$-glucan $/ 30 \mathrm{~g}$ avCHO reduced glucose iAUC by an average of $27 \pm 3 \mathrm{mmol} \times \mathrm{min} / \mathrm{L}$ relative to a variety of different comparators. However, the clinical relevance of this difference depends on the population studied and the nature of the comparator. For example, blood glucose iAUC in 77 subjects without diabetes varied from $\sim 80$ to $\sim 550 \mathrm{mmol} \times \mathrm{min} / \mathrm{L}$ after consuming $50 \mathrm{~g}$ glucose, and $\sim 40$ to $\sim 450 \mathrm{mmol} \times \mathrm{min} / \mathrm{L}$ after $50 \mathrm{~g}$ avCHO from white bread [11]. Thus, a $27 \mathrm{mmol} \times \mathrm{min} / \mathrm{L}$ difference in iAUC is equivalent to reductions varying from 5-34\% relative to glucose or 6-68\% relative to white bread. Assessing the relative differences within trials and considering the nature of the comparator test-meal may provide more precise and clinically meaningful estimates of the effect of OBG on PPGR.
For these reasons, we aimed to synthesize the evidence from acute, crossover, single-meal, controlled feeding trials of the effect of OBG on postprandial glucose and insulin responses in humans regardless of health status, and to explore whether OBG dose, MW, nature of the comparator, health status, OBG food form, study methodology quality, duration of follow-up and risk of bias modified these effects.

\section{Methods}

This systematic review and meta-analysis was conducted according to the Cochrane Handbook for Systematic Reviews of Interventions [12]. Data were reported in accordance with the Preferred Reporting Items for Systematic Reviews and Meta-Analyses (PRISMA) guidelines [13]. The study protocol was registered on the Open Science Forum (OSF) registry [14].

\section{Data sources}

MEDLINE, EMBASE, and the Cochrane Central Register of Controlled Trials were searched through October 27, 2020. Electronic searches were supplemented with manual searches of references from included studies. The detailed search strategy is outlined in Supplementary Table 1.

\section{Study selection}

We included randomized and non-randomized acute, crossover, single-meal, controlled feeding trials that investigated the effects of OBG or oat bran (high in OBG) added to a carbohydrate-containing meal in humans regardless of health status on at least one of the following 4 variables: glucose and insulin incremental area-under-the-curve (iAUC) and incremental peak-rise (iPeak). To be included, the comparator (control) test-meal had to contain an equivalent amount of avCHO as the OBG-containing testmeal; however, the sources of avCHO did not have to be the same. In some studies, the sources of avCHO in the control and the OBG test-meals were comparable (matched control; e.g., OBG-containing spaghetti vs OBG-free spaghetti) and in some they were different (unmatched control; e.g., OBGcontaining spaghetti vs white bread). We excluded trials of parallel design, chronic feeding, studies in which participants were not fasting at baseline, and trials that did not provide appropriate outcome data, used non-oat sources of $\beta$-glucan (e.g., BBG), and used oats or oat flour as the sole source of OBG. We excluded non-oat sources of $\beta$-glucan because, as explained above, their structure and effect on glycaemic responses differs from that of OBG. We excluded studies using oats or oat flour as the sole source of OBG 
because each gram of OBG in oats or oat flour is accompanied by about $13 \mathrm{~g}$ avCHO which itself influences glucose and insulin responses in ways which are affected by cooking and processing [8] and, thus, confounds the effect of OBG in potentially unpredictable ways.

\section{Data extraction}

Two investigators (AZ and JCN) independently reviewed and extracted relevant data from each included report. Extracted data included participant characteristics (e.g., health status, age, sex, BMI), OBG dose (expressed as $\mathrm{g}$ OBG $/ 30 \mathrm{~g}$ avCHO [5]), OBG MW $(<300 \mathrm{~kg} / \mathrm{mol}=$ low; 300 to $<1000 \mathrm{~kg} / \mathrm{mol}=$ medium; $\geq 1000 \mathrm{~kg} / \mathrm{mol}=$ high), intervention and comparator meal characteristics (nature of foods [e.g. glucose, bread, muffins, pasta, juice] and macronutrient composition [total carbohydrate, total fibre, soluble fibre, available carbohydrate (avCHO), protein, fat]), study design, duration of follow-up, setting, funding sources, and outcome data. In the absence of numerical values for outcome data and the inability to contact study authors, values were extracted from figures using Plot Digitizer, version 2.5.1 (Free Software Foundation, Boston, MA). If outcome data were provided for multiple follow-up durations (e.g., $120 \mathrm{~min}$ and $180 \mathrm{~min}$ ) in a single study, the data points for the 120 mins were preferred to minimize heterogeneity. Similarly, matched control comparisons were preferred to unmatched control comparisons when available. The same two investigators also assessed risk of bias and study methodology quality from each included report. Risk of bias was evaluated using version 2 of the Cochrane risk-of-bias (RoB 2) tool, where bias was assessed in five distinct domains (bias arising from the randomization process, bias due to deviations from intended interventions, bias due to missing outcome data, bias in measurement of the outcome, and bias in selection of the reported result). Within each domain, the investigators answered one or more signalling questions and these answers led to judgements of "low risk of bias", "some concerns", or "high risk of bias" $[10,15]$. Study methodology quality was assessed for each trial comparison based on eleven predetermined criteria outlined in Supplementary Table 2 which was adapted from a previous study $[11,16]$. This assessment identified protocol components that have been established as significant determinants of the accuracy and precision of PPGR measurements [17-28]. The score was used to inform the responses to the signalling questions in the "bias in measurement of the outcome" domain of the RoB 2 tool and to determine the influence of study methodology quality in the relationship between OBG and PPGR. One of the criteria (criteria \#10) was specific to iAUC methodology and was excluded for iPeak outcomes. Studies were categorized as having high methodology quality and a low risk of bias in measurement of the outcome if they specified the OBG dose and met $\geq 7$ or $\geq 6$ of the other criteria for iAUC and iPeak outcomes, respectively. Studies which did not meet these thresholds were considered to have low methodology quality and some concerns for risk of bias in the measurement of the outcome. Any discrepancies in data extraction, risk of bias, and study methodology quality assessments were reconciled by consensus with a third reviewer (TMSW).

\section{Outcomes}

The primary outcome was glucose iAUC. Secondary outcomes were insulin iAUC, glucose iPeak and insulin iPeak.

\section{Data synthesis and analysis}

The pooled effect estimate for each outcome was expressed as a ratio of means (RoM) with $95 \%$ confidence intervals (CIs). RoM is a method to present continuous measures on a ratio scale and is calculated by dividing the mean value in the intervention group by the mean value in the control group (Supplementary Fig. 1). This method facilitates clinical interpretation (e.g., RoM of 1.2 indicates an increase of $20 \%$ in the intervention group compared to the control group; a RoM of 0.7 indicates a reduction of 30\%) and controls for baseline differences in the comparator groups across studies [29-31]. Paired analyses were applied to all comparisons $[31,32]$. If multiple comparisons were available in the same population, we controlled for unit of analysis error by dividing the $\mathrm{N}$ of the respective arm by the number of times it was included.

STATA Version 16 (StataCorp, TX, USA) was used to conduct all analyses. Natural log-transformed RoM (ln $[\mathrm{RoM}]$ ) and standard error (SE) of the $\ln [\mathrm{RoM}]$ were pooled using the generic inverse variance method with DerSimonian and Laird random effects models [33, 34]. Fixed effects models were only used if fewer than 5 trials were present for an outcome [35]. Linear dose-response was modelled using one-stage random effects with restricted maximum likelihood methods assuming a linear function [36]. Non-linear dose response was modelled with restricted cubic splines with three knots. The Wald's test was used to examine departure from linearity.

Inter-study heterogeneity was assessed using the Cochran $\mathrm{Q}$ statistic and quantified using the $\mathrm{I}^{2}$ statistic, where $I^{2} \geq 50 \%$ and $P_{\mathrm{Q}}<0.10$ were considered evidence of substantial heterogeneity [12]. Potential sources of heterogeneity were investigated by sensitivity analyses and subgroup analyses. For determination of whether a single trial comparison exerted undue influence, sensitivity analyses were performed in which we recalculated the pooled effect estimates and heterogeneity after removing each 
individual trial. Studies whose removal explained the heterogeneity, changed the direction or significance of the effect, or altered the effect size by $10 \%$ or more were considered influential. We conducted a priori subgroup and publication bias analyses for all relationships with $\geq 10$ trial comparisons. A priori and post hoc categorical subgroup analyses were conducted using meta-regression with a $P<$ 0.05 indicative of a significant difference between subgroups. A priori subgroups included health status, OBG dose, comparator, food form, duration of follow-up, risk of bias, and study methodology quality. By mistake, MW was not included in the registered list of subgroups, and, since this was not discovered until after the initial analysis had been completed, we cannot prove that examination of the effect of MW was done a priori; therefore it has to be considered as a post hoc subgroup analysis. Publication bias was assessed by visual inspection of funnel plots for asymmetry and formal testing by the Egger's and Begg's tests $[37,38]$. If evidence of publication bias was detected, Duval and Tweedie nonparametric "trim and fill" analyses were applied to assess the effect of the imputed "missing" studies [39].

\section{Grading of the evidence}

The overall certainty of the evidence was evaluated using the GRADE tool where the certainty of the evidence was graded as high, moderate, low, or very low certainty [40-42]. Randomized controlled trials are graded as high certainty evidence by default and then downgraded on the basis of the following pre-specified criteria: risk of bias (weight of studies shows important risk of bias as assessed by the RoB2 tool), inconsistency (substantial unexplained interstudy heterogeneity, $I^{2} \geq 50 \%$ and $P_{\mathrm{Q}}<0.10$ ), indirectness (presence of factors that limit generalizability of the results), imprecision, and publication bias (significant evidence of small-study effects). For the purposes of GRADE we considered the results to be imprecise if the $95 \%$ CIs of the pooled effect estimates were wide or overlapped 0.8 (equivalent to a $20 \%$ reduction in glucose iAUC which Health Canada considered to be the minimum physiologically relevant difference [43]). Certainty of the evidence was upgraded if a dose-response was detected.

\section{Results}

\section{Search results}

We identified a total of 1522 reports, of which 1353 were excluded based on title and/or abstract review (Supplementary Fig. 2). The remaining 169 articles were reviewed in full and 135 were excluded. A total of 35 reports
Table 1 Summary of trial characteristics.

\begin{tabular}{|c|c|}
\hline \multicolumn{2}{|l|}{ Characteristic $^{\mathrm{a}}$} \\
\hline Trial comparisons & 103 \\
\hline Participants & 538 \\
\hline Follow-up duration, minutes & $120(60-240)$ \\
\hline \multicolumn{2}{|l|}{ Participant characteristics } \\
\hline - Age, years & $37(22-66)$ \\
\hline - Male:female ${ }^{\mathrm{b}}(\%)$ & $50: 50$ \\
\hline - BMI, $\mathrm{kg} / \mathrm{m}^{2}$ & $24.9(20.6-31.1)$ \\
\hline \multicolumn{2}{|l|}{ Health Status, \# of trial comparisons } \\
\hline • Healthy & 77 \\
\hline - Type 2 Diabetes & 11 \\
\hline - Overweight & 9 \\
\hline - Mixed (Health \& Overweight) & 5 \\
\hline - Metabolic Syndrome & 1 \\
\hline \multicolumn{2}{|l|}{ Intervention characteristics } \\
\hline - OBG dose, g & $4.2(0.2-11.7)$ \\
\hline - OBG dose, $\mathrm{g} / 30 \mathrm{~g}$ available carbohydrates & $2.8(0.1-22.6)$ \\
\hline \multicolumn{2}{|l|}{ - Food source, \# of trials } \\
\hline ○ OBG-enriched/oat bran muffins & 23 \\
\hline o OBG/oat bran added to glucose/dextrose & 20 \\
\hline o OBG-enriched/oat bran cereal & 13 \\
\hline $\begin{array}{l}\circ \text { OBG-enriched/oat bran added to oatmeal } \\
\text { porridge }\end{array}$ & 13 \\
\hline o Oat granola/muesli with oat bran flakes & 10 \\
\hline ○ OBG-enriched bread & 8 \\
\hline ○ OBG-enriched/oat bran snack bar or product & 7 \\
\hline $\begin{array}{l}\text { ○ OBG-enriched/oat bran beverage (juice, } \\
\text { shake, drink) }\end{array}$ & 7 \\
\hline o Oat bran pasta & 2 \\
\hline - Available carbohydrate, g & $50(13-75)$ \\
\hline \multicolumn{2}{|l|}{ Comparator characteristics } \\
\hline \multicolumn{2}{|l|}{ • Type } \\
\hline ○ Matched & 66 \\
\hline ○ Unmatched & 37 \\
\hline \multicolumn{2}{|l|}{ - Food source, \# of trial comparisons } \\
\hline ○ Glucose/dextrose/maltodextrin solution & 32 \\
\hline$\circ$ White bread & 21 \\
\hline ○ Muffin & 16 \\
\hline $\begin{array}{l}\text { Wheat porridge or oatmeal without } \\
\text { added OBG }\end{array}$ & 12 \\
\hline$\circ$ Wheat granola/muesli with cornflakes & 7 \\
\hline ○ Snack bar/product & 5 \\
\hline ○ Cornflakes cereal & 4 \\
\hline ○ Juice/shake/drink & 4 \\
\hline$\circ$ Durum wheat pasta & 2 \\
\hline - Available carbohydrate, g & $50(13-75)$ \\
\hline \multicolumn{2}{|l|}{ Setting, \# of trial comparisons } \\
\hline - North America & 70 \\
\hline - Europe & 25 \\
\hline
\end{tabular}


Table 1 (continued)

\begin{tabular}{ll}
\hline Characteristic $^{\text {a }}$ & \\
\hline - South America & 4 \\
- Australia & 3 \\
- Asia & 1 \\
Funding source, \# of trial comparisons & \\
- Agency & 43 \\
- Industry & 28 \\
- Agency \& Industry & 9 \\
- Not reported & 23 \\
\hline
\end{tabular}

$O B G$ oat $\beta$-glucan, $B M I$ body mass index.

${ }^{a}$ Median (range) of mean data, unless otherwise indicated.

$\mathrm{b}_{30 / 31}$ studies provided data on sex.

${ }^{c}$ Agency funding is that from government, university, or not-for-profit sources. Industry funding is that from trade organizations that obtain revenue from the sale of products.

[6, 7, 44-76], containing data for 103 trial comparisons involving 538 participants, met the eligibility criteria and were included in the final analyses.

\section{Trial characteristics}

Table 1 and Supplementary Table 3 show the summary and individual characteristics of all included trials and trial comparisons. The median follow-up duration across all trials was $120 \mathrm{~min}$ (range 60-240). All trials were in outpatient settings with the majority conducted in North America (68\%) and Europe (24\%). Trial funding came from agency sources $(42 \%)$, industry sources $(27 \%)$, or both (9\%), with no funding information reported in $22 \%$ of the trials. Participants were males and females (50\% male, 50\% female) aged (median (range) of the reported means) 37 (22-66) years with a BMI of $24.9(20.6-31.1) \mathrm{kg} / \mathrm{m}^{2}$. Most trials were conducted in healthy participants $(75 \%)$ with some in individuals with type 2 diabetes (11\%), those who were overweight (9\%), both healthy and overweight (5\%), or had metabolic syndrome $(1 \%)$.

The interventions involved consumption of OBG added to various foods including muffins (22\%), glucose/ dextrose solutions and gels (19\%), cereals (13\%), porridges $(13 \%)$, muesli $(10 \%)$, bread $(8 \%)$, snack bars or products (7\%), juices/shakes/drinks (7\%) and pasta (2\%). Four food form categories were identified: gels, liquids, semi-solids (hot cereals) and solids (bread, muffins, food bars and granola). A median (range) of $4.2(0.2-11.7) \mathrm{g}$ OBG was added to a median 50 (13-75) $\mathrm{g}$ avCHO, for a median OBG dose per $30 \mathrm{~g}$ avCHO of $2.8(0.1-22.6) \mathrm{g}$. The comparators in these trials included glucose/dextrose/maltodextrin solutions $(31 \%)$, white bread $(20 \%)$, muffins $(16 \%)$, porridge or oatmeal $(12 \%)$, granola or muesli with cornflakes (7\%), snack bars $(5 \%)$, cornflakes cereal (4\%), juice/shakes/drinks (4\%), and pasta (2\%) without added OBG.

\section{Risk of bias}

Supplementary Tables 4-7 show individual risk of bias assessments. Low study methodology quality was identified in $23 \%, 41 \%, 3 \%$ and $15 \%$ of trial comparisons for iAUC glucose and insulin and iPeak glucose and insulin, respectively, and were assessed as having some concerns for risk of bias in the measurement of the outcome. Supplementary Figs. 3-6 show summary risk of bias assessments. Most trials included for glucose iAUC and iPeak were assessed as having low overall risk of bias whereas, $71 \%$ of trials for insulin iAUC either had some concerns or high overall risk of bias and $69 \%$ of trials for iPeak insulin had some concerns for overall risk of bias.

\section{Effect of oat $\beta$-glucan on glucose iAUC}

In 98 trial comparisons involving 508 participants, pooled analysis showed that OBG reduced glucose iAUC by $23 \%$ (RoM 0.77 [95\% CI 0.74, 0.81], $p<0.001$ with substantial heterogeneity, $I^{2}=59.9 \%, P_{\mathrm{Q}}<0.001$ ) (Fig. 1A). Removal of individual trials did not alter the direction, significance, or magnitude ( $10 \%$ or more) of the effect or the evidence for heterogeneity. There was significant effect modification by comparator $\left(p=0.034\right.$, residual $\left.I^{2}=58.0 \%, P_{\mathrm{Q}}<0.001\right)$, OBG dose $\left(p<0.001\right.$, residual $\left.I^{2}=7.2 \%, P_{\mathrm{Q}}=0.284\right)$, and OBG MW $\left(p=0.004\right.$, residual $\left.I^{2}=38.6 \%, P_{\mathrm{Q}}=0.004\right)$, (Fig. 1A, Supplementary Figs. 7-9). Only OBG doses $>1.5 \mathrm{~g} / 30 \mathrm{~g}$ avCHO and $\mathrm{MW}>300 \mathrm{~kg} / \mathrm{mol}$ were shown to significantly reduce glucose iAUC. Duration was a significant effect modifier ( $p=0.021$, residual $I^{2}=58.3 \%, P_{\mathrm{Q}}$ $<0.001$ ) with the largest reduction seen for a postprandial period of $240 \mathrm{~min}$ which was limited to one study with 3 trial comparisons (Supplementary Fig. 10A). Food form was also a significant effect modifier $(p=0.045$, residual $\left.I^{2}=60.1 \%, P_{\mathrm{Q}}<0.001\right)$ with the effect of liquid food forms being significantly less than solid $(p=0.01)$ and semi-solid $(p=0.02)$ (Supplementary Fig. 10A).

No significant effect modifications were observed by health status $\left(p=0.35\right.$, residual $\left.I^{2}=59 \%, P_{\mathrm{Q}}<0.001\right)$, study methodology quality ( $p=0.226$, residual $I^{2}=59.9 \%$, $\left.P_{\mathrm{Q}}<0.001\right)$ and overall risk of bias $(p=0.61$, residual $\left.I^{2}=60 \%\right)($ Fig. 1A, Supplementary Figs. 10A, 11-12).

A significant linear dose-response relationship was observed suggesting an $8 \%$ reduction in glucose iAUC per $1 \mathrm{~g} \mathrm{OBG} / 30 \mathrm{~g}$ avCHO (slope 0.92 [95\% CI 0.91, 0.94], $p<0.001$ ) (Fig. 2A and Supplementary Fig. 13). When this relationship was assessed based on MW (Fig. 3A-C), 


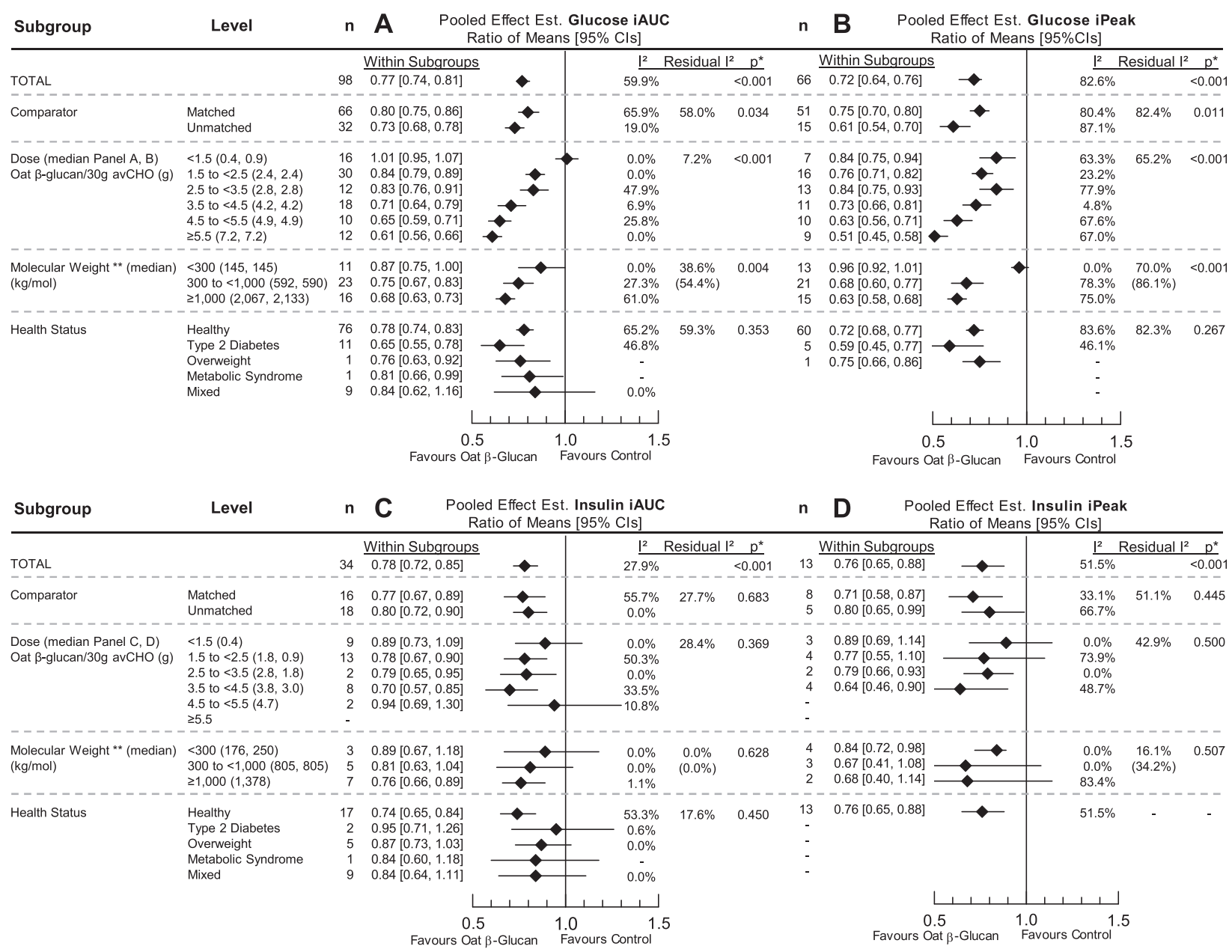

Fig. 1 Effect of oat $\beta$-glucan on postprandial glycaemic and insulinaemic responses. Pooled effect estimates of the effect of oat $\beta$-glucan on the incremental area under the curve (iAUC) for blood glucose (A), incremental peak rise (iPeak) for blood glucose (B), iAUC insulin (C), and iPeak insulin (D). Pooled effect estimates are expressed as ratios of means (RoMs, black diamond) with 95\% CIs (solid lines). Pooled analyses were conducted using the generic inverse variance method with random effects models. Interstudy heterogeneity was tested by the Cochran Q statistic $\left(\chi^{2}\right)$ at a significance level of $P_{\mathrm{Q}}<0.10$ (not shown) and quantified by $\mathrm{I}^{2}$. The residual $\mathrm{I}^{2}$ value represents unexplained heterogeneity for each subgroup. n, number of trial comparisons. *Differences between subgroups were tested using meta-regression and the significance level was reported as a $p$ value, where $p<0.05$ was considered significant. medium and high MW OBG demonstrated significant doseresponse relationships $(p<0.001)$ whereas, low MW OBG did not $(p=0.052)$. The dose-response with high MW OBG was more precise than with medium MW OBG (slope 0.92 [0.91, 0.93] vs. 0.93 [0.91, 0.96], respectively). Supplementary Figs. 14 and 15 show the dose-response relationship by health status and study methodology quality, respectively. More precise dose-response relationships were observed in trials assessing healthy individuals (slope 0.93 [0.92, 0.94]) compared to those with type 2 diabetes (slope 0.91 [0.86, 0.97]), and in trials of high study methodology quality (slope $0.93[0.91,0.94])$ compared to those with low study methodology quality (slope 0.91 $[0.86,0.96])$. The slope of the dose-response relationship was greater but less precise for the unmatched comparators $(0.90[0.86,0.93])$ than the matched comparators $(0.94$
$[0.92,0.96])$ but the confidence intervals overlapped (Supplementary Fig. 16).

\section{Effect of Oat $\beta$-glucan on glucose iPeak}

In 66 trial comparisons involving 313 participants, pooled analysis showed that OBG reduced glucose iPeak by $28 \%$ $(0.72[0.64,0.76], p<0.001$, with substantial heterogeneity, $I^{2}=82.6 \%, P_{\mathrm{Q}}<0.01$ ) (Fig. 1B). Removal of individual trials did not alter the direction, significance, or magnitude ( $10 \%$ or more) of the effect or the evidence for heterogeneity. There was significant effect modification by the comparator $\left(p<0.011\right.$, residual $\left.I^{2}=82.4 \%, P_{\mathrm{Q}}<0.001\right)$, OBG dose $\left(p<0.001\right.$, residual $\left.I^{2}=65.2 \%, P_{\mathrm{Q}}<0.001\right)$ and OBG MW $\left(p<0.001\right.$, residual $\left.\mathrm{I}^{2}=70.0 \%, P_{\mathrm{Q}}<0.001\right)$ (Fig. 1B, Supplementary Figs. 17-19). Greater reductions in 

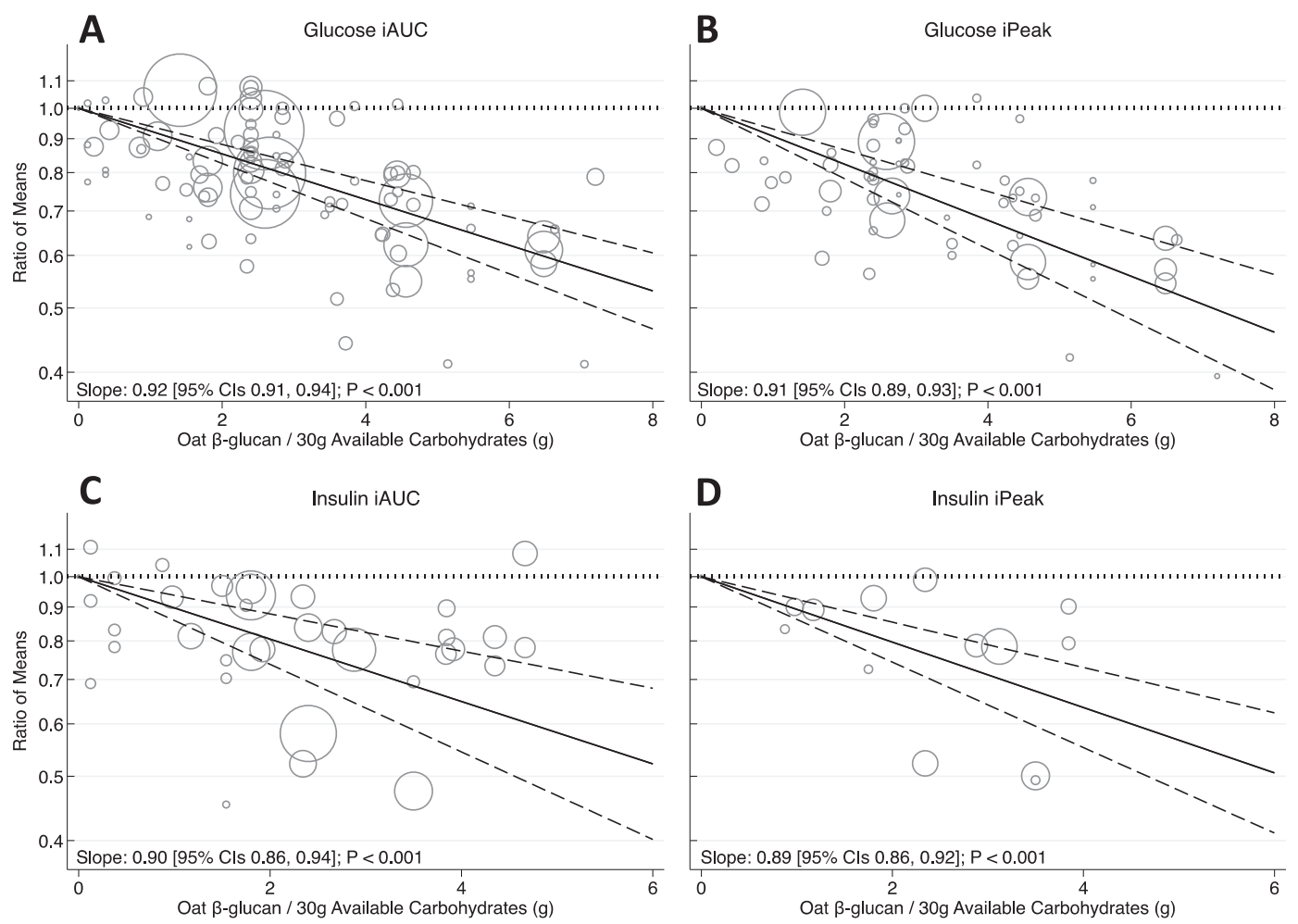

Fig. 2 Effect of oat $\beta$-glucan dose on postprandial glycaemic and insulinaemic responses. Pooled dose-response relationship between oat $\beta$-glucan and incremental area under the curve (iAUC) for blood glucose (A), incremental peak rise (iPeak) for blood glucose (B), iAUC insulin (C), and iPeak insulin (D). Changes in the outcomes (yaxis) are presented as ratios of means (RoMs). Oat $\beta$-glucan dose is presented on a $1 \mathrm{~g} / 30 \mathrm{~g}$ available carbohydrate scale. Individual

comparisons are represented by the circles, with the weight of the study in the overall analysis represented by the size of the circles. The central straight line represents the fitted dose response estimate with outer dashed lines representing the $95 \%$ confidence intervals (CIs), which was modelled using one-stage random effects with the generic inverse variance and restricted maximum likelihood methods, assuming a linear function.

glucose iPeak were observed when OBG containing foods were compared to unmatched comparators than with matched comparators. OBG MW $>300 \mathrm{~kg} / \mathrm{mol}$ led to greater reductions in glucose iPeak compared to $\mathrm{MW}<300 \mathrm{~kg} /$ mol. Duration was a significant effect modifier $(p=0.024$, residual $\left.I^{2}=82.8 \%, P_{\mathrm{Q}}<0.001\right)$ with the largest reduction seen for a postprandial period of $240 \mathrm{~min}$ which was limited to one study with 3 trial comparisons (Supplementary Fig. 10B). Food form was also a significant effect modifier $\left(p=0.029\right.$, residual $\left.I^{2}=82.8 \%, P_{\mathrm{Q}}<0.001\right)$ with a larger reduction observed in meals where OBG was consumed in semi-solid versus liquid form (Supplementary Fig. 10B). No significant effect or heterogeneity modifications were observed by health status $(p=0.267$, residual $\left.I^{2}=82.3 \%, \quad P_{\mathrm{Q}}<0.001\right)$, study methodology quality $\left(p=0.347\right.$, residual $\left.\mathrm{I}^{2}=82.8 \%, \mathrm{P}_{\mathrm{Q}}<0.001\right)$, and overall risk of bias $\left(p=0.15\right.$, residual $\left.I^{2}=82 \%\right)$ (Fig. $1 \mathrm{~B}$, Supplementary Figs. 10B, 20-21).

A significant linear dose-response relationship was observed suggesting a 9\% reduction in glucose iPeak per $1 \mathrm{~g} \mathrm{OBG} / 30 \mathrm{~g}$ avCHO (slope 0.91 [95\% CI 0.89, 0.93], $p<0.001$ ) (Fig. 2B and Supplementary Fig. 13). When this relationship was assessed based on MW (Fig. 3D-F), medium and high MW OBG demonstrated significant doseresponse relationships $(p<0.001)$ whereas, low MW OBG did not $(p=0.108)$. Supplementary Figs. 14-16 show the dose-response relationship by health status, study methodology quality, and the comparator. More precise doseresponse relationships were observed in trials assessing healthy individuals (slope $0.91[0.89,0.94]$ ) compared to those with type 2 diabetes (slope 0.90 [0.83, 0.97]), in high study methodology quality trials (slope $0.91[0.89,0.93]$ ) compared to low study methodology quality trials (slope $0.86[0.76,0.98]$ ), and matched comparators (slope 0.92 $[0.90,0.94])$ compared to unmatched comparators (slope $0.89[0.85,0.93])$.

\section{Effect of Oat $\beta$-glucan on insulin iAUC}

In 34 trial comparisons involving 231 participants, pooled analysis showed that OBG reduced insulin iAUC by $22 \%$ (0.78 [0.72, 0.85], $p<0.001$, with no evidence of 

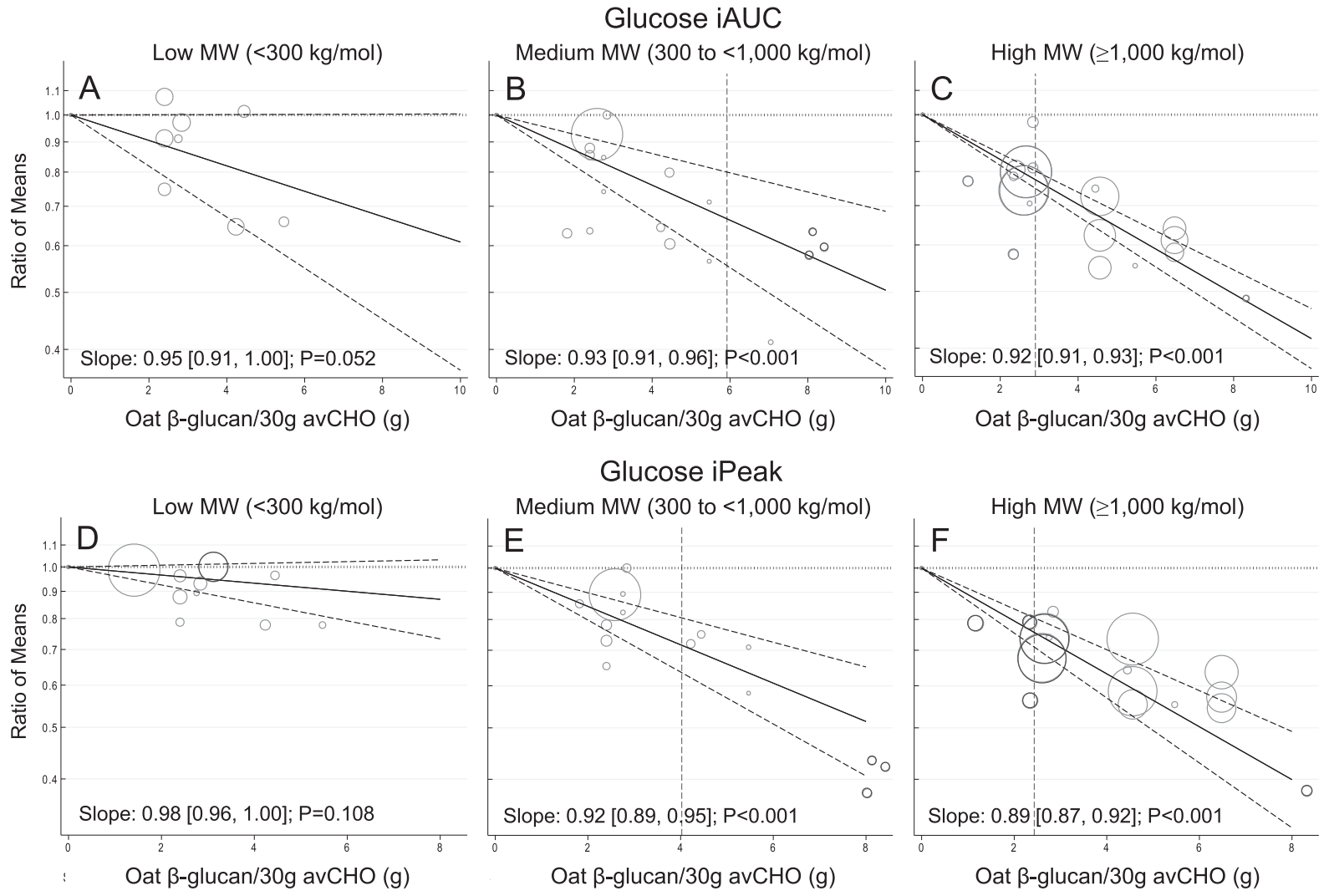

Fig. 3 Effect of oat $\beta$-glucan molecular weight and dose on postprandial glycaemic responses. Pooled dose-response relationship by low, medium and high molecular weight oat $\beta$-glucan on the incremental area under the curve (iAUC) for blood glucose (A-C) and incremental peak rise (iPeak) for blood glucose (D-F). Changes in the outcomes (y-axis) are presented as ratios of means (RoMs). Oat $\beta$-glucan dose is presented on a $1 \mathrm{~g} / 30 \mathrm{~g}$ available carbohydrate scale. Individual comparisons are represented by the circles, with the weight of the study in the overall analysis represented by the size of the circles.

heterogeneity, $I^{2}=27.9 \%, P_{\mathrm{Q}}=0.10$ ) (Fig. 1C). Removal of individual trials did not alter the direction, significance, or magnitude (10\% or more) of the effect or the evidence for heterogeneity. No significant effect modifications were observed by dose, MW, comparator, health status, food form, duration, study methodology quality, and risk of bias (Fig. 1C, Supplementary Figs. 10C, 22-26).

A significant linear dose-response relationship was observed suggesting a $10 \%$ reduction in insulin iAUC per $1 \mathrm{~g} \mathrm{OBG} / 30 \mathrm{~g}$ avCHO (slope 0.90 [95\% CI 0.86, 0.94], $p<0.001$ ) (Fig. 2C and Supplementary Fig. 13). Health status (not illustrated due to limited data in type 2 diabetes population) and the comparator did not modify the doseresponse relationship (Supplementary Fig. 16). A larger dose-response relationship was observed in trials of low study methodology quality (slope $0.82[0.77,0.87]$ ) compared to trials of high study methodology quality (slope $0.92[0.88,0.96])$ (Supplementary Fig. 15).
The light grey and blue circles represent the trial comparisons with matched and unmatched comparators, respectively. The central straight line represents the fitted dose response estimate with outer dashed lines representing the $95 \%$ confidence intervals (CIs), which was modelled using one-stage random effects with the generic inverse variance and restricted maximum likelihood methods, assuming a linear function. The vertical dashed line represents the dose level at which the upper-bound $95 \%$ CIs cross the physiologically relevant threshold of a $20 \%$ reduction.

\section{Effect of Oat $\beta$-glucan on insulin iPeak}

In 13 trial comparisons involving 115 participants, pooled analysis showed that OBG reduced insulin iPeak by $24 \%$ $(0.76$ [0.65, 0.88], $p<0.001$, with no evidence of heterogeneity, $I^{2}=51.5 \%, P_{\mathrm{Q}}=0.11$ ) (Fig. 1D). Removal of individual trials did not alter the direction, significance, or magnitude (10\% or more) of the effect or the evidence for heterogeneity. No significant effect modifications were observed by dose, MW, comparator, food form, duration, study methodology quality, and risk of bias (Supplementary Figs. 10D, 27-30). Subgroup analysis by health status was not conducted as data were limited to only healthy individuals (Supplementary Fig. 31).

A significant linear dose-response relationship was observed suggesting a $11 \%$ reduction in insulin iAUC per $1 \mathrm{~g} \mathrm{OBG} / 30 \mathrm{~g}$ avCHO (slope 0.89 [95\% CI 0.86, 0.92], $p<$ 0.001) (Fig. 2D and Supplementary Fig. 13). A significant 
dose-response relationship was observed in trials of high methodology quality (slope $0.89[0.86,0.93]$ ) whereas trials of low study methodology quality did not demonstrate a significant dose-response relationship (slope 0.95 [0.82, 1.10]) (Supplementary Fig. 15). The dose-response relationship was similar when assessed by the comparator (Supplementary Fig. 16).

\section{Publication bias analyses}

Visual inspection of funnel plots for publication bias showed no evidence of asymmetry or small-study effects for glucose and insulin iAUC and iPeak (Supplementary Fig. 32). Both Egger and Begg tests were non-significant for all outcomes.

\section{GRADE assessment}

A summary of the GRADE assessments for each outcome is shown in Supplementary Table 8. Our certainty in the evidence was high for the effect of $\mathrm{OBG}$ on reducing glucose and insulin iAUC and iPeak. We identified serious imprecision in the insulin iAUC and iPeak pooled effect estimates, as the upper bounds of the $95 \%$ Cis overlapped the minimally important difference of 0.8 , which could not be explained by our subgroup analyses. There was evidence of imprecision of the glucose iAUC pooled effect estimate, however subgroup analyses illustrated precise pooled effect estimates at doses $\geq 3.5 \mathrm{~g} / 30 \mathrm{~g}$ available carbohydrates of OBG and from high MW OBG and thus, no downgrade was applied. We also identified serious inconsistency in the glucose iPeak due to significant unexplained heterogeneity $\left(I^{2}=82.6 \%\right)$. Substantial heterogeneity of the pooled effect estimate was observed in glucose iAUC $\left(I^{2}=59.9 \%\right)$, however, the between-study variance was largely explained by dose (residual $I^{2}=7.2 \%, P_{\mathrm{Q}}=0.284$ ) and partially by molecular weight (residual $I^{2}=38.6 \%, P_{Q}=0.004$ ). Although $71 \%$ of trials for insulin iAUC either had some concerns or high overall risk of bias, and $69 \%$ of trials for iPeak insulin had some concerns for overall risk of bias, subgroup analyses suggest that risk of bias was not a significant effect modifier of the pooled effect estimates and thus, no downgrades were applied. We upgraded our certainty in the evidence for glucose and insulin iAUC and iPeak due to the presence of dose-response relationships.

\section{Discussion}

\section{Summary of findings}

This systematic review and meta-analysis of acute, crossover, single-meal, controlled feeding trials, including 103 comparisons in 538 participants from 35 reports, showed that OBG reduced glucose and insulin iAUC by $23 \%$ and $22 \%$, respectively, and glucose and insulin iPeak by $28 \%$ and $24 \%$, respectively. OBG dose, MW, the comparator, intervention food form, and study duration were significant effect modifiers of the reduction in glucose iAUC and iPeak.

Significant linear dose-responses were found for all endpoints, with each $\mathrm{g} \mathrm{OBG} / 30 \mathrm{~g}$ avCHO reducing glucose iAUC by (mean $[95 \% \mathrm{CI}]) 8[6,9] \%$, glucose iPeak by 9 $[7,11] \%$, insulin iAUC by $10[6,14] \%$ and insulin iPeak by $11[8,14] \%$. However, the dose-response relationships for glycaemic response became steeper and had smaller 95\% CI as OBG MW increased; for OBG with $\mathrm{MW}<300 \mathrm{~kg} / \mathrm{mol}$, 300 to $<1000 \mathrm{~kg} / \mathrm{mol}$ and $\geq 1000 \mathrm{~kg} / \mathrm{mol}$, respectively, each g OBG/30 g avCHO reduced glucose iAUC by $5[9,0] \%, 7$ $[4,9] \%$ and $8[7,9] \%$ and glucose iPeak by $2[4,0] \%, 8$ $[5,11] \%$ and $11[8,13] \%$. All outcomes were similar in participants with and without diabetes.

\section{Results in relation to previous studies}

Our findings are consistent with an earlier review by Tosh [1] which showed that OBG elicited a dose-dependent reduction in glucose iAUC and also significantly reduced glucose iPeak and insulin iAUC. However, Tosh expressed the differences in iAUC as absolute values ( $\mathrm{mmol} \times \mathrm{min} / \mathrm{L})$, excluded treatments where the $\beta$-glucan MW had been deliberately reduced to $<250 \mathrm{~kg} / \mathrm{mol}$ and excluded studies in individuals with diabetes. Our results also show that OBG significantly reduced glycaemic and insulinaemic responses, but expressing the results as RoM rather than absolute values allows the magnitude and clinical utility of the effects to be determined, and allows for valid comparison of the effects in subjects with and without diabetes. Furthermore, by including in our analysis studies using low MW OBG we were able to determine how much low MW OBG reduces glucose and insulin responses in comparison to the effects of medium and high MW OBG.

In substantiating the health claim for $\mathrm{OBG}$ and $\mathrm{BBG}$ on the reduction of PPGR, the EFSA panel [5] provided a narrative review of 6 studies, 2 of which we included $[54,57]$ and 4 of which we did not because the sources of $\beta$-glucan were oat flour or barley [77-80]. The basis for the panel's opinion that “...the studies above show an effect of oat and barley beta-glucans in decreasing post-prandial glycaemic responses ... at doses of at least $4 \mathrm{~g}$ per $30 \mathrm{~g}$ available carbohydrates" is difficult to appreciate because information about the number of comparisons, the dose of $\beta$-glucan and endpoint used (e.g., iPeak, iAUC) is not provided. Our pooled result from 98 comparisons is similar to the EFSA conclusion in suggesting that a dose of $>3.5 \mathrm{~g}$ $\mathrm{OBG} / 30 \mathrm{~g}$ avCHO is required to obtain a $\geq 20 \%$ reduction in glucose iAUC. However, our results suggest that if high 
MW OBG is used, $\geq 20 \%$ reductions in glucose iAUC and iPeak can be obtained with 2.9 and $2.5 \mathrm{~g}$ OBG $/ 30 \mathrm{~g}$ avCHO, respectively. Furthermore, based on 5 studies in participants with diabetes considered by the EFSA panel (only 3 of which we included $[47,56,69]$ since the other 2 were not acute test meal studies [81, 82]), the EFSA panel noted that "the evidence provided does not establish that results obtained in patient populations treated with antidiabetic medications can be generalized to the target population with respect to postprandial glycaemic responses" [4]. The rationale for this conclusion is not clear, but our results show that $\mathrm{OBG}$ reduces glycaemic responses to a similar, or perhaps even greater, extent in individuals with type 2 diabetes than in those without diabetes, respectively, for glucose iAUC (35\% vs. 22\%) and glucose iPeak (41\% vs. $28 \%$ ). This is consistent with the finding that the relative glycaemic responses of foods compared to glucose (i.e., their glycaemic index values) are similar in subjects with and without diabetes [83].

\section{Effect modifiers and other sources of heterogeneity}

Effect modifiers of the effect of OBG on glucose response were OBG dose, MW, the comparator, intervention food form, and study duration, but there were no significant effect modifiers for insulin response.

\section{OBG dose and MW}

The mechanism by which viscous fibres reduce postprandial glucose and insulin responses is thought to be related to their ability to increase the viscosity of the contents of the gastrointestinal tract (GIT) [84]. The viscosity of OBG solutions is determined by the concentration and MW of the OBG [85]. This is consistent with our finding that both dose and MW significantly modify the effect of OBG on glycaemic response. However, in order to increase the viscosity of the contents of the GIT, the OBG consumed has to be released from the food matrix. The solubility, or bioavailability, of OBG in foods varies [7, 65, 66] and reducing OBG solubility reduces its effect on glycaemic responses [60]. Thus, unmeasured variation in OBG solubility may contribute to the unaccounted for heterogeneity of our results. Furthermore, the viscosity of glucose solutions containing OBG is not always related to their glycaemic impact [59] because the concentration, and hence viscosity, of OBG solutions within the stomach may be reduced by gastric fluid secretions [86]. High viscosity could reduce glycaemic responses by delaying gastric emptying or reducing the rate of digestion and absorption of carbohydrates in the small intestine, or both; however, the exact mechanism is not completely understood. For example, we recently showed that consuming $4 \mathrm{~g}$ OBG in a breakfast test-meal reduced both the rate of gastric emptying and the glycaemic response, effects which were abrogated by reducing the dose of $\mathrm{OBG}$ or by reducing its MW [76]. Nevertheless, there was no correlation between gastric emptying and glycaemic response elicited by the 4 test meals within the 28 subjects.

\section{Comparator}

The effect of OBG on glucose responses was greater for unmatched vs matched Control test-meals. The nature of the comparator is important because source of avCHO, quantified by glycaemic-index (the extent to which the avCHO in a food raises glucose iAUC relative to an equal weight of glucose), is an independent determinant of glucose iAUC [87]. For test-meals containing equivalent amounts of avCHO, protein and fat, differences in glycaemic-index are proportional to differences in glucose iAUC [88]. In 26 $(81 \%)$ of the 32 unmatched comparisons the source of avCHO in the Control test-meals was glucose, dextrose, maltodextrin or white bread, with the source in the other 6 being cream of rice, wheat muffin or cornflakes. If the glycaemic-index of the avCHO in an OBG test-meal is less than that of its Control, the difference in glucose iAUC would be larger than if their glycaemic-indices had been equivalent. The estimated mean glycaemic-index of the unmatched Control test-meals, 81 , was $14 \%$ greater than that of the OBG test-meals, 71, a difference which could account for the $10 \%$ lower mean ROM for the unmatched vs matched comparators 0.73 vs 0.80 .

\section{Intervention food form}

OBG in liquid form had a smaller effect on glycaemic response than in semi-solid or solid food forms. It seems unlikely this is due to food form per se, since, if anything, an equivalent amount of OBG in liquid form, vs solid form, may be more soluble in the gut and, hence, have a greater effect on glycaemic response. The effect modification due to food form is more likely accounted for by the lower MW of the OBG contained in the liquid vs the semi-solid and solid forms. If an OBG-enriched liquid is to remain a palatable liquid, the OBG must be hydrolyzed to reduce its MW and viscosity, whereas high MW OBG can be incorporated into palatable semi-solid and solid foods. This is likely why none of the liquid test meals contained high-MW OBG. The percentage of low-, medium- and high-MW OBG contained in the $n=11$ liquid forms $(45 \%, 55 \%, 0 \%$, respectively) differed significantly from that in 11 semisolid $(9 \%, 55 \%, 36 \%, p=0.036)$ and the 26 solid forms $(19 \%, 35 \%, 46 \%, p=0.020)$, with the distribution in semisolid and solid forms being similar. An estimate of the effect of MW in the different forms can be obtained by 
multiplying the mean $\%$ reduction for low-, medium- and high-MW OBG $(13 \%, 25 \%$ and $32 \%$, respectively, for glucose iAUC) by the respective proportion of low-, medium- and high-MW OBG within each form and summing the products (e,g., the expected iAUC reduction for liquids = $0.13 \times 0.45+0.25 \times 0.55+0.32 \times 0=0.20)$. The expected reductions for glucose iAUC, 20\%, 26\% and $26 \%$ for liquid, semi-solid and solid, respectively, are similar to those observed, $14 \%, 22 \%, 25 \%$, as are those for glucose iPeak, $19 \%, 31 \%, 29 \%$, vs $22 \%, 37 \%, 22 \%$.

\section{Study duration}

Although study duration was an effect modifier, sensitivity analyses showed that the difference was attributed to the one study with a $4 \mathrm{~h}$ postprandial duration. It is known that the method used to calculate iAUC, the duration of blood sampling and the interval between blood samples can influence glucose iAUC [17, 89] and iPeak [20]. Furthermore, the method used by authors to calculate iAUC is often not indicated, and the likelihood of incorrect calculation may be $>50 \%$ [26]. These factors may contribute towards unexplained heterogeneity in the results.

\section{Clinical implications}

In synthesizing the available data on the effect of OBG on acute glycaemic responses, the question arises as to whether reducing acute glucose and insulin responses has any clinical relevance. Reducing the glycaemic impact of high carbohydrate meals with treatments which reduce the rate of carbohydrate absorption, such as $\alpha$-glucosidase inhibitors $[90,91]$ and low glycaemic index foods [92] have desirable physiological effects for many people, particularly for those with pre-diabetes or diabetes. Our findings suggest that the acute effects of OBG on glucose and insulin responses in subjects without diabetes can be extrapolated to people with diabetes. Whether the acute effect of OBG on postprandial glucose response translates into clinically meaningful benefits in long-term glycaemic control is not clear. An earlier systematic review and meta-analysis of 4 randomized controlled trials in 350 individuals with type 2 diabetes found that $\mathrm{OBG}$ consumption of $2.5-3.5 \mathrm{~g} /$ day significantly lowered $\mathrm{HbA}_{1 \mathrm{c}}$ by $0.21 \%$ and fasting plasma glucose by $0.52 \mathrm{mmol} / \mathrm{L}$, without affecting fasting plasma insulin concentrations [93]. However, an updated analysis showed no effect on HbA1c (mean difference, $-0.55 \%$ [95\% CI $-1.21,0.11])$ and fasting glucose $(-0.54 \mathrm{mmol} / \mathrm{L}[-1.70$, $0.62]$ ) when data were pooled from 5 trials in 535 individuals with type 2 diabetes [94]. Furthermore, the mechanism by which OBG improved glycaemic control in these studies may not be related to an effect on acute glycaemic response but rather to an ability of OBG to favourably alter the colonic microbiome [95]. There is a need for more longterm randomized controlled trials to confirm the effect of OBG on glycaemic control in diabetes and determine a mechanism of action.

If the acute effect of $\mathrm{OBG}$ on postprandial glucose responses is beneficial, how large of an effect is required for physiological relevance? Health Canada opined that the minimum physiologically relevant difference in glucose iAUC is $20 \%$ [43]. We used this conservative value to assess whether there was imprecision of the results as an indicator of certainty of the evidence. However, differences in diet glycaemic index of 10-15\% may be clinically relevant. For example, in a randomized clinical trial of 210 subjects with type 2 diabetes with baseline HbA1c of $7.1 \%$ studied for 6 months, a $14 \%$ reduction in diet GI was associated with a clinically meaningful reduction of HbA1c of $0.32 \%$ relative to control $(p<0.001)[96,97]$. Our results suggest that only $\sim 2 \mathrm{~g} / 30 \mathrm{~g}$ avCHO of high MW OBG is required to reduce glucose iAUC by $14 \%$ with $95 \%$ certainty (Fig. 3C).

\section{Limitations}

The pooled effect estimates were imprecise for insulin iAUC and iPeak, as the $95 \%$ CIs overlap the minimally important difference for clinical benefit. This imprecision may be due to the limited range of doses included. Although the dose response for each outcome showed that the $95 \%$ CIs of the regressions entered the bounds of the minimally important difference as the dose increased, the categorical dose response analysis illustrated imprecision across all dose ranges. Therefore, more studies, including interventions with doses $>4.5 \mathrm{~g} / 30 \mathrm{~g}$ available carbohydrate of OBG, may improve the precision of the effect of OBG on insulin outcomes. There was also substantial heterogeneity in the overall pooled effect estimate for glucose iPeak, that could only be partially explained by ranges in dose and molecular weight of the included trials.

Of note, our health status category was based on population demographics described in the included studies, therefore it is possible that there may be some overlap between the healthy and overweight categories, in which populations that were categorized as healthy likely also included overweight individuals. Data were also limited in individuals with type 2 diabetes for insulin iAUC and not available for insulin iPeak and thus, more studies would be useful to improve the precision of our findings in this population.

\section{Conclusion}

Our synthesis of the available evidence from acute, crossover, single-meal, controlled feeding trials demonstrates 
that $\mathrm{OBG}$ leads to a clinically meaningful reduction in postprandial glucose responses provided that a sufficient amount of high MW OBG is provided. OBG interventions also resulted in clinically meaningful but imprecise reductions in insulin iAUC and iPeak, for which dose may modify the magnitude of the effect. Health status, OBG food form, postprandial duration, study methodology quality and risk of bias did not meaningfully modify these effects. More studies are needed to improve precision in the effects of $\mathrm{OBG}$ in diabetes and to explore whether the acute reductions in glycaemic response translate into clinically meaningful benefits in long-term glycaemic control. These conclusions apply to the addition of purified OBG or oat products highly enriched in OBG to food. Although OBG is present in oats, it is unclear if our findings can be extrapolated to commercial foods containing oats as a source of $\mathrm{OBG}$ due to the presence of other nutrients (avCHO, protein and fat) and differences in food processing which may modify these effects.

Funding This work was supported by INQUIS Clinical Research, Ltd. and by an unrestricted grant from PepsiCo, Inc (Category 1). This study was designed and carried out by INQUIS Clinical Research, Ltd. PepsiCo, Inc. was not involved in the study hypothesis/design, execution, analysis, or interpretation.

Author contributions AZ assisted with study design, conducted the systematic search, screened eligible studies, extracted data, conducted meta-analysis, interpreted results and assisted in drafting the manuscript. JCN assisted with study design, screened eligible studies, extracted and analyzed data, interpreted results, and drafted the manuscript. TAK assisted with the data analysis and interpretation and editing the manuscript. JLS assisted with data interpretation and editing the manuscript. TMSW conceived the project, assisted with study design, assisted in drafting the manuscript, approved the final version and takes overall responsibility for the content of the manuscript. All the authors had access to the data and reviewed and approved the final manuscript.

\section{Compliance with ethical standards}

Conflict of interest AZ and JCN are employees of INQUIS Clinical Research, Ltd. TAK has received research support from the Canadian Institutes of Health Research (CIHR), the International Life Science Institute (ILSI), and National Honey Board. He has been an invited speaker at the Calorie Control Council Annual meeting for which he has received an honorarium. JLS has received research support from the Canadian Foundation for Innovation, Ontario Research Fund, Province of Ontario Ministry of Research and Innovation and Science, Canadian Institutes of health Research (CIHR), Diabetes Canada, PSI Foundation, Banting and Best Diabetes Centre (BBDC), American Society for Nutrition (ASN), INC International Nut and Dried Fruit Council Foundation, National Dried Fruit Trade Association, The Tate and Lyle Nutritional Research Fund at the University of Toronto, The Glycemic Control and Cardiovascular Disease in Type 2 Diabetes Fund at the University of Toronto (a fund established by the Alberta Pulse Growers), and the Nutrition Trialists Fund at the University of Toronto (a fund established by an inaugural donation from the Calorie Control Council). He has received in-kind food donations to support a randomized controlled trial from the Almond Board of California,
California Walnut Commission, American Peanut Council, Barilla, Unilever, Unico/Primo, Loblaw Companies, Quaker, Kellogg Canada, and WhiteWave Foods. He has received travel support, speaker fees and/or honoraria from Diabetes Canada, Mott's LLP, Dairy Farmers of Canada, FoodMinds LLC, International Sweeteners Association, Nestlé, Pulse Canada, Canadian Society for Endocrinology and Metabolism (CSEM), GI Foundation, Abbott, Biofortis, ASN, Northern Ontario School of Medicine, INC Nutrition Research \& Education Foundation, European Food Safety Authority (EFSA), Comité Européen des Fabricants de Sucre (CEFS), and Physicians Committee for Responsible Medicine. He has or has had ad hoc consulting arrangements with Perkins Coie LLP, Tate \& Lyle, and Wirtschaftliche Vereinigung Zucker e.V. He is a member of the European Fruit Juice Association Scientific Expert Panel and Soy Nutrition Institute (SNI) Scientific Advisory Committee. He is on the Clinical Practice Guidelines Expert Committees of Diabetes Canada, European Association for the study of Diabetes (EASD), Canadian Cardiovascular Society (CCS), and Obesity Canada. He serves or has served as an unpaid scientific advisor for the Food, Nutrition, and Safety Program (FNSP) and the Technical Committee on Carbohydrates of the International Life Science Institute (ILSI) North America. $\mathrm{He}$ is a member of the International Carbohydrate Quality Consortium (ICQC), Executive Board Member of the Diabetes and Nutrition Study Group (DNSG) of the EASD, and Director of the Toronto 3D Knowledge Synthesis and Clinical Trials foundation. His wife is an employee of AB InBev. TMSW is an employee and part owner of INQUIS Clinical Research, Ltd.

Publisher's note Springer Nature remains neutral with regard to jurisdictional claims in published maps and institutional affiliations.

Open Access This article is licensed under a Creative Commons Attribution 4.0 International License, which permits use, sharing, adaptation, distribution and reproduction in any medium or format, as long as you give appropriate credit to the original author(s) and the source, provide a link to the Creative Commons license, and indicate if changes were made. The images or other third party material in this article are included in the article's Creative Commons license, unless indicated otherwise in a credit line to the material. If material is not included in the article's Creative Commons license and your intended use is not permitted by statutory regulation or exceeds the permitted use, you will need to obtain permission directly from the copyright holder. To view a copy of this license, visit http://creativecommons. org/licenses/by/4.0/.

\section{References}

1. Tosh SM. Review of human studies investigating the postprandial blood-glucose lowering ability of oat and barley food products. Eur J Clin Nutr. 2013;67:310-7.

2. Whitehead A, Beck EJ, Tosh S, Wolever TM. Cholesterollowering effects of oat $\beta$-glucan: a meta-analysis of randomized controlled trials. Am J Clin Nutr. 2014;100:1413-21.

3. Ho HV, Sievenpiper JL, Zurbau A, Blanco Mejia A, Jovanovski E, Au-Yeung F, et al. The effect of oat $\beta$-glucan on LDL-cholesterol, non-HDL-cholesterol and apoB for CVD risk reduction: a systematic review and meta-analysis of randomised-controlled trials. Br J Nutr. 2016;116:1369-82.

4. Wang Y, Harding SV, Thandapilly SJ, Tosh SM, Jones PJH, Ames NP. Barley $\beta$-glucan reduces blood cholesterol levels via interrupting bile acid metabolism. Brit J Nutr. 2017;118:822-9.

5. EFSA Panel on Dietetic Products, Nutrition and Allergies (NDA Scientific Opinion on the substantiation of health claims related to beta-glucans from oats and barley and maintenance of normal 
blood LDL-cholesterol concentrations (ID 1236, 1299), increase in satiety leading to a reduction in energy intake (ID 851, 852), reduction of post-prandial glycaemic responses (ID 821, 824), and "digestive function" (ID 850) pursuant to Article 13(1) of Regulation (EC) No 1924/2006. EFSA J. 2011;9:2207 https://doi.org/ 10.2903/j.efsa.2011.2207.21 pp.accessed on May 18, 2020.

6. Wolever TMS, Jenkins AL, Prudence K, Johnson J, Duss R, Chu Y. Effect of adding oat bran to instant oatmeal on glycaemic response in humans - a study to establish the minimum effective dose of oat beta-glucan. Food Funct. 2018;9:1692-1700.

7. Tosh SM, Brummer Y, Wolever TMS, Wood PJ. Glycemic Response to Oat Bran Muffins Treated to Vary Molecular Weight of $\beta$-Glucan. Cereal Chem. 2008;85:211-7.

8. Tosh SM, Chu Y. Systematic review of the effect of processing of whole-grain oat cereals on glycaemic response. $\mathrm{Br} \mathrm{J}$ Nutr. 2015;114:1256-62.

9. Aldughpassi A, Abdel-Aal EM, Wolever TMS. Barley cultivar, kernel composition and processing affect the glycemic index. J Nutr. 2012;142:1666-71.

10. Cloetens L, Ulmius M, Johansson-Persson A, Åkesson B, Önning G. Role of dietary beta-glucans in the prevention of the metabolic syndrome. Nutr Rev. 2012;70:444-58.

11. Wolever TMS, Jenkins AL, Vuksan V, Campbell J. The glycaemic index values of foods containing fructose are affected by metabolic differences between subjects. Eur J Clin Nutr. 2009;63:1106-14.

12. Higgins JPT, Green S, Eds. Cochrane Handbook for systematic reviews of interventions version 5.1.0 [updated March 2011]. The Cochrane Collaboration; 2011. http://handbook.cochrane.org (Accessed May 18, 2020).

13. Moher D, Liberati A, Tetzlaff J, Altman DG. Preferred reporting items for systematic reviews and meta-analyses: the PRISMA statement. PLoS Med 2009;6:e1000097.

14. Wolever, T (2020, January 29). Oat beta-glucan and postprandial glycemia outcomes: a systematic review and meta-analysis of acute feeding trials. Retrieved from: https://osf.io/gmc4y.

15. Sterne JAC, Savović J, Page MJ, Elbers RG, Blencowe NS, Boutron I, et al. RoB 2: a revised tool for assessing risk of bias in randomised trials. Brit Med J. 2019;366:14898.

16. Wolever TM, Giddens JL, Sievenpiper JL. Effect of ethnicity on glycaemic index: a systematic review and meta-analysis. Nutr Diabetes. 2015;5:e170.

17. Wolever TM. Effect of blood sampling schedule and method of calculating the area under the curve on validity and precision of glycaemic index values. Br J Nutr. 2004;91:295-301.

18. Velangi A, Fernandes G, Wolever TM. Evaluation of a glucose meter for determining the glycemic responses of foods. Clin Chim Acta 2005;356:191-8.

19. Wolever TMS, Ip B, Moghaddam E. Measuring glycaemic responses: duplicate fasting samples or duplicate measures of one fasting sample? Br J Nutr. 2006;96:799-802.

20. Lui JL, Lan-Pidhainy X, Brummer Y, Tosh SM, Wood PJ, Wolever TM. Effect of blood sampling schedule on the ability to discriminate between postprandial glycemic responses. Nutrition. 2009;25:1064-6.

21. Aldughpassi A, Wolever TM. Effect of coffee and tea on the glycaemic index of foods: no effect on mean but reduced variability. Br J Nutr. 2009;101:1282-5.

22. Young KWH, Wolever TMS. Effect of volume and type of beverage consumed with a standard test meal on postprandial blood glucose responses. Nutr Res. 1998;18:1857-63.

23. Wolever TM, Bolognesi C. Source and amount of carbohydrate affect postprandial glucose and insulin in normal subjects. J Nutr. 1996;126:2798-806.

24. Wolever TMS, Gibbs AL, Spolar M, Hitchner EV, Heimowitz C. Equivalent glycemic load (EGL): a method for quantifying the glycemic responses elicited by low carbohydrate foods. Nutr Metab (Lond). 2006;3:33.

25. Lee BM, Wolever TM. Effect of glucose, sucrose and fructose on plasma glucose and insulin responses in normal humans: comparison with white bread. Eur J Clin Nutr. 1998;52:924-8.

26. Wolever TM, Brand-Miller JC, Abernethy J, Astrup A, Atkinson F, Axelsen $\mathrm{M}$, et al. Measuring the glycemic index of foods: interlaboratory study. Am J Clin Nutr. 2008;87:247s-257s.

27. Wolever T, Bolognesi C. Time of day influences relative glycaemic effect of foods. Nutr Res. 1996;16:381-4.

28. Campbell JE, Glowczewski T, Wolever TMS. Controlling subjects' prior diet and activities does not reduce within-subject variation of postprandial glycemic responses to foods. Nutr Res. 2003;23:621-9.

29. Friedrich JO, Adhikari NKJ, Beyene J. The ratio of means method as an alternative to mean differences for analyzing continuous outcome variables in meta-analysis: a simulation study. BMC Med Res Methodol. 2008;8:32.

30. Friedrich JO, Adhikari NKJ, Beyene J. Ratio of means for analyzing continuous outcomes in meta-analysis performed as well as mean difference methods. J Clin Epidemiol. 2011;64:556-64.

31. Lajeunesse MJ. On the meta-analysis of response ratios for studies with correlated and multi-group designs. Ecology. 2011;92:2049-55.

32. Elbourne DR, Altman DG, Higgins JP, Curtin F, Worthington $\mathrm{HV}$, Vail A. Meta-analyses involving cross-over trials: methodological issues. Int J Epidemiol. 2002;31:140-9.

33. DerSimonian R, Laird N. Meta-analysis in clinical trials. Control Clin Trials. 1986;7:177-88.

34. Deeks JJ HJ, Altman DG (editors). Chapter 10: Analysing data and undertaking meta-analyses. In: Higgins JPT, Thomas J, Chandler J, Cumpston M, Li T, Page MJ, Welch VA (editors). Cochrane Handbook for Systematic Reviews of Interventions version 6.0 (updated July 2019). Cochrane, 2019. Available from www.training.cochrane.org/handbook.

35. Tufanaru C, Munn Z, Stephenson M, Aromataris E. Fixed or random effects meta-analysis? Common methodological issues in systematic reviews of effectiveness. Int J Evid Based Health. 2015;13:196-207.

36. Orsini N, Li R, Wolk A, Khudyakov P, Spiegelman D. Metaanalysis for linear and nonlinear dose-response relations: examples, an evaluation of approximations, and software. Am J Epidemiol. 2012;175:66-73.

37. Egger M, Davey Smith G, Schneider M, Minder C. Bias in metaanalysis detected by a simple, graphical test. Brit Med J. 1997;315:629-34

38. Begg CB, Mazumdar M. Operating characteristics of a rank correlation test for publication bias. Biometrics. 1994;50:1088-101.

39. Duval S, Tweedie R. Trim and fill: A simple funnel-plot-based method of testing and adjusting for publication bias in metaanalysis. Biometrics. 2000;56:455-63.

40. GRADEpro GDT: GRADEpro Guideline Development Tool [Software]. McMaster University, 2015 (developed by Evidence Prime, Inc.). gradepro.org.

41. Schünemann H, Brożek J, Guyatt G, Oxman A, editors. GRADE handbook for grading quality of evidence and strength of recommendations. Updated October 2013. The GRADE Working Group, 2013. www.guidelinedevelopment.org/handbook.

42. Santesso N, Glenton C, Dahm P, Garner P, Akl EA, Alper B, et al. GRADE guidelines 26: informative statements to communicate the findings of systematic reviews of interventions. J Clin Epidemiol. 2020;119:126-35.

43. Bureau of Nutritional Sciences, Food Directorate, Health Products and Food Branch, Health Canada. Summary of Health Canada's Assessment of a Health Claim about a Polysaccharide Complex 
(Glucomannan, Xanthan Gum, Sodium Alginate) and a Reduction of the Post-Prandial Blood Glucose Response. 2016. https://www. canada.ca/content/dam/hc-sc/migration/hc-sc/fn-an/alt_formats/ pdf/label-etiquet/claims-reclam/assess-evalu/glucose-complexpolysaccharides-complexe-glycemique-eng.pdf (Accessed May 18, 2020).

44. Beck EJ, Tosh SM, Batterham MJ, Tapsell LC, Huang XF. Oat beta-glucan increases postprandial cholecystokinin levels, decreases insulin response and extends subjective satiety in overweight subjects. Mol Nutr Food Res. 2009;53:1343-51.

45. Behall KM, Scholfield DJ, Hallfrisch JG, Liljeberg-Elmstahl HG. Consumption of both resistant starch and beta-glucan improves postprandial plasma glucose and insulin in women. Diabetes Care. 2006;29:976-81.

46. Braaten JT, Wood PJ, Scott FW, Riedel KD, Poste LM, Collins MW. Oat gum lowers glucose and insulin after an oral glucose load. Am J Clin Nutr. 1991;53:1425-30.

47. Braaten JT, Scott FW, Wood PJ, Riedel KD, Wolynetz MS, Brulé D, et al. High beta-glucan oat bran and oat gum reduce postprandial blood glucose and insulin in subjects with and without type 2 diabetes. Diabet Med. 1994;11:312-8.

48. Brennan MA, Derbyshire EJ, Brennan CS, Tiwari BK. Impact of dietary fibre-enriched ready-to-eat extruded snacks on the postprandial glycaemic response of non-diabetic patients. Mol Nutr Food Res. 2012;56:834-7.

49. Brummer Y, Duss R, Wolever TMS, Tosh SM. Glycemic response to extruded oat bran cereals processed to vary in molecular weight. Cereal Chem. 2012;89:255-61.

50. Galvão Cândido F, Silva Ton WT, Gonçalves Alfenas Rde C. Addition of dietary fiber sources to shakes reduces postprandial glycemia and alters food intake. Nutr Hosp. 2014;31:299-306.

51. Granfeldt Y, Nyberg L, Bjorck I. Muesli with $4 \mathrm{~g}$ oat beta-glucans lowers glucose and insulin responses after a bread meal in healthy subjects. Eur J Clin Nutr. 2008;62:600-7.

52. Hartvigsen ML, Gregersen S, Lærke HN, Holst JJ, Bach Knudsen $\mathrm{KE}$, Hermansen K. Effects of concentrated arabinoxylan and betaglucan compared with refined wheat and whole grain rye on glucose and appetite in subjects with the metabolic syndrome: a randomized study. Eur J Clin Nutr. 2014;68:84-90.

53. Hlebowicz J, Darwiche G, Bjorgell O, Almer LO. Effect of muesli with $4 \mathrm{~g}$ oat beta-glucan on postprandial blood glucose, gastric emptying and satiety in healthy subjects: a randomized crossover trial. J Am Coll Nutr. 2008;27:470-5.

54. Holm J, Bjorck I. Bioavailability of starch in various wheat-based bread products: evaluation of metabolic responses in healthy subjects and rate and extent of in vitro starch digestion. Am J Clin Nutr. 1992;55:420-9.

55. Holm J, Koellreutter B, Wursch P. Influence of sterilization, drying and oat bran enrichment of pasta on glucose and insulin responses in healthy subjects and on the rate and extent of in vitro starch digestion. Eur J Clin Nutr. 1992;46:629-40.

56. Jenkins AL, Jenkins DJ, Zdravkovic U, Wursch P, Vuksan V. Depression of the glycemic index by high levels of beta-glucan fiber in two functional foods tested in type 2 diabetes. Eur J Clin Nutr. 2002;56:622-8.

57. Juntunen KS, Niskanen LK, Liukkonen KH, Poutanen KS, Holst JJ, Mykkanen HM. Postprandial glucose, insulin, and incretin responses to grain products in healthy subjects. Am J Clin Nutr. 2002;75:254-62.

58. Kwong MG, Wolever TM, Brummer Y, Tosh SM. Attenuation of glycemic responses by oat beta-glucan solutions and viscoelastic gels is dependent on molecular weight distribution. Food Funct. 2013;4:401-8.

59. Kwong MG, Wolever TM, Brummer Y, Tosh SM. Increasing the viscosity of oat beta-glucan beverages by reducing solution volume does not reduce glycaemic responses. $\mathrm{Br} \mathrm{J}$ Nutr. 2013;110:1465-71.

60. Lan-Pidhainy X, Brummer Y, Tosh SM, Wolever TM, Wood PJ. Reducing beta-glucan solubility in oat bran muffins by freezethaw treatment attenuates its hypoglycemic effect. Cereal Chem. 2007;84:512-7.

61. Lindström C, Voinot A, Forslund A, Holst O, Rascón, Öste R, et al. An oat bran-based beverage reduce postprandial glycaemia equivalent to yoghurt in healthy overweight subjects. Int J Food Sci Nutr. 2015;66:700-5.

62. Panahi S, Ezatagha A, Temelli F, Vasanthan T, Vuksan V. Betaglucan from two sources of oat concentrates affect postprandial glycemia in relation to the level of viscosity. J Am Coll Nutr. 2007;26:639-44.

63. Panahi S, Ezatagha A, Jovanovski E, Jenkins A, Temelli F, Vasanthan T, et al. Glycemic effect of oat and barley beta-glucan when incorporated into a snack bar: a dose escalation study. J Am Coll Nutr. 2014;33:442-9.

64. Paquin J, Bédard A, Lemieux S, Tajchakavit S, Turgeon SL. Effects of juices enriched with xanthan and beta-glucan on the glycemic response and satiety of healthy men. Appl Physiol Nutr Metab. 2013;38:410-4.

65. Regand A, Tosh SM, Wolever TM, Wood PJ. Physicochemical properties of beta-glucan in differently processed oat foods influence glycemic response. J Agric Food Chem. 2009;57:8831-8.

66. Regand A, Chowdhury Z, Tosh SM, Wolever TMS, Wood P. The molecular weight, solubility and viscosity of oat beta-glucan affect human glycemic response by modifying starch digestibility. Food Chem. 2011;129:297-304.

67. Rieder A, Knutsen SH, Sainz Fernandez A, Ballance S. At a high dose even partially degraded beta-glucan with decreased solubility significantly reduced the glycaemic response to bread. Food Funct. 2019;10:1529-39.

68. Tapola N, Karvonen H, Niskanen L, Mikola M, Sarkkinen E. Glycemic responses of oat bran products in type 2 diabetic patients. Nutr Metab Cardiovasc Dis. 2005;15:255-61.

69. Tappy L, Gügolz E, Würsch P. Effects of breakfast cereals containing various amounts of beta-glucan fibers on plasma glucose and insulin responses in NIDDM subjects. Diabetes Care. 1996;19:831-4.

70. Ulmius M, Johansson-Persson A, Krogh M, Olsson P, Önning G. An oat bran meal influences blood insulin levels and related gene sets in peripheral blood mononuclear cells of healthy subjects. Genes Nutr. 2011;6:429-39.

71. Wood PJ, Braaten JT, Scott FW, Riedel D, Poste LM. Comparisons of viscous properties of oat and guar gum and the effects of these and oat bran on glycemic index. J Agric Food Chem. 1990;38:753-7.

72. Wood PJ, Braaten JT, Scott FW, Riedel KD, Wolynetz MS, Collins MW. Effect of dose and modification of viscous properties of oat gum on plasma glucose and insulin following an oral glucose load. Br J Nutr. 1994;72:731-43.

73. Amiruddin NEN, Zahary MN, Bhaskar R, Mhd Jalil AM. Glycaemic index, palatability and acceptability of energy drinks prepared with $\beta$-glucan and whey protein. Food Res. 2020;4:421-30.

74. Binou P, Yanni AE, Stergiou A, Karavasilis K, Konstantopoulos P, Perrea D, et al. Enrichment of bread with beta-glucans or resistant starch induces similar glucose, insulin and appetite hormone responses in healthy adults. Eur J Nutr 2020 May. https:// doi.org/10.1007/s00394-020-02265-6. Epub ahead of print.

75. Wang L, Ye F, Feng L, Wei F, Zhao G. The effects of oat $\beta$-glucan incorporation on the quality, structure, consumer acceptance and glycaemic response of steamed bread. J Texture Stud. 2017;48:562-70. 
76. Wolever TMS, Tosh SM, Spruill SE, Jenkins AL, Ezatagha A, Duss $\mathrm{R}$, et al. Increasing oat $\beta$-glucan viscosity in a breakfast meal slows gastric emptying and reduces glycemic and insulinemic responses but has no effect on appetite, food intake, or plasma ghrelin and PYY responses in healthy humans: a randomized, placebo-controlled, crossover trial. Am J Clin Nutr. 2020;11:319-28.

77. Behall KM, Scholfield DJ, Hallfrisch J. Comparison of hormone and glucose responses of overweight women to barley and oats. $\mathrm{J}$ Am Coll Nutr. 2005;24:182-8.

78. Liljeberg HG, Granfeldt YE, Björck IM. Products based on a high fiber barley genotype, but not on common barley or oats, lower postprandial glucose and insulin responses in healthy humans. $\mathrm{J}$ Nutr. 1996;126:458-66.

79. Östman E, Rossi E, Larsson H, Brighenti F, Björck I. Glucose and insulin responses in healthy men to barley bread with different levels of $(1 \rightarrow 3 ; 1 \rightarrow 4)$ - $\beta$-glucans; predictions using fluidity measurements of in vitro enzyme digests. J Cereal Sci. 2006;43:230-5.

80. Yokoyama WH, Hudson CA, Knuckles BE, Chiu MM, Sayre RN, Turnlund JR, et al. Effect of barley $\beta$-glucan in durum wheat pasta on human glycemic response. Cereal Chem. 1997;74:293-6.

81. Pick ME, Hawrysh ZJ, Gee MI, Toth E, Garg ML, Hardin RT. Oat bran concentrate bread products improve long-term control of diabetes: a pilot study. J Am Diet Assoc. 1996;96:1254-61.

82. Pick ME, Hawrysh ZJ, Gee MI, Toth E. Barley Bread products improve glycemic control of type 2 subjects. Int J Food Sci Nutr. 1998;49:71-78

83. Lan-Pidhainy X, Wolever TM. Are the glycemic and insulinemic index values of carbohydrate foods similar in healthy control, hyperinsulinemic and type 2 diabetic patients? Eur J Clin Nutr. 2011;65:727-34

84. Jenkins DJ, Wolever TM, Leeds AR, Gassull MA, Dilawari JB, Goff DV, et al. Dietary fibres, fibre analogues, and glucose tolerance: importance of viscosity. Br Med J. 1978;1:1392-4.

85. Wood PJ, Beer MU, Butler G. Evaluation of role of concentration and molecular weight of oat beta-glucan in determining effect of viscosity on plasma glucose and insulin following an oral glucose load. Br J Nutr. 2000;84:19-23.

86. Steinert RE, Raederstorff D, Wolever TM. Effect of consuming oat bran mixed in water before a meal on glycemic responses in healthy humans-a pilot study. Nutrients. 2016;8:524.
87. Kochan AM, Wolever TMS, Chetty VT, Anand SS, Gerstein HC, Sharma AM. Glycemic index predicts individual glucose responses after self-selected breakfasts in free-living abdominally obese adults. J Nutr. 2012;142:27-32.

88. Wolever TMS. Is glycaemic index (GI) a valid measure of carbohydrate quality? Eur J Clin Nutr. 2013;67:522-31.

89. Gannon MC, Nuttall JA. Factors affecting interpretation of postprandial glucose and insulin areas. Diabetes Care. 1987;10:759-63.

90. van de Laar FA, Lucassen PL, Akkermans RP, van de Lisdonk EH, Rutten GE, van Weel C. Alpha-glucosidase inhibitors for patients with type 2 diabetes: results from a Cochrane systematic review and meta-analysis. Diabetes Care. 2005;28:154-63.

91. Moelands SV, Lucassen PL, Akkermans RP, De Grauw WJ, Van de Laar FA. Alpha-glucosidase inhibitors for prevention or delay of type 2 diabetes mellitus and its associated complications in people at increased risk of developing type 2 diabetes mellitus. Cochrane Database Syst Rev. 2018;12:Cd005061.

92. Thomas DE, Elliott EJ. The use of low-glycaemic index diets in diabetes control. Br J Nutr. 2010;104:797-802.

93. Shen XL, Zhao T, Zhou Y, Shi X, Zou Y, Zhao G. Effect of oat $\beta$-glucan intake on glycaemic control and insulin sensitivity of diabetic patients: a meta-analysis of randomized controlled trials. Nutrients. 2016;8:39.

94. Jovanovski E, Khayyat R, Zurbau A, Komishon A, Mazhar N, Sievenpiper JL. et al. Should viscous fiber supplements be considered in diabetes control? Results from a systematic review and meta-analysis of randomized controlled trials. Diabetes Care.2019;42:755-66.https://doi.org/10.2337/dc19-er08a.

95. Rebello CJ, Burton J, Heiman M, Greenway FL. Gastrointestinal microbiome modulator improves glucose tolerance in overweight and obese subjects: a randomized controlled pilot trial. J Diabetes Complications. 2015;29:1272-6.

96. Jenkins DJ, Kendall CW, McKeown-Eyssen G, Josse RG, Silverberg J, Booth GL, et al. Effect of a low-glycemic index or a high-cereal fiber diet on type 2 diabetes: a randomized trial. JAMA. 2008;300:2742-53.

97. Center for Drug Evaluation and Research. Guidance for industry: diabetes mellitus: developing drugs and therapeutic biologics for treatment and prevention (DRAFT GUIDANCE). Silver Spring, MD, USA: U.S. Department of Health and Human Services Food and Drug Administration; 2008. p. 1-30. 